\title{
Propuesta de un modelo para analizar y medir la percepción de la calidad de servicio en los programas de cuarto nivel
}

\section{Proposal of a model in order to analyze and measure the perception of the quality of service in fourth level programs}

Galo Antonio Molina Mármol

Mayra Liuviana Vega Chica

Universidad Espíritu Santo, Ecuador

Autor para correspondencia: gmolinam@uees.edu.ec, mvegach@uees.edu.ec

Fecha de recepción: 04 de Enero 2017 - Fecha de aceptación: 10 de Junio de 2018

Resumen: El presente artículo tiene como objetivo proponer un modelo para analizar la percepción y eficacia de la calidad de servicio de los estudiantes en programas de cuarto nivel en el Ecuador, actualmente las instituciones gubernamentales han aumentado sus reglamentos para evaluar los establecimientos que ofrecen una educación superior. La metodología que se tomó para realizar este trabajo fue la de observación, se revisaron varios artículos de autores que se centraron en elaborar diferentes tipos de modelos enfocados a la evaluación de la calidad de servicio de las instituciones educativas existentes como: Serqual, servperf y DIHEQS. El modelo que se propone es "Calidad percibida en posgrado" (CPEP). Este modelo está compuesto por las siguientes dimensiones: a) Profesores y enseñanza; b) Malla curricular; c) Tecnología y comunicación; d) Actividades adicionales; y d) Administración y organización, que se dirige a la lealtad y convirtiéndose en una ventaja competitiva hacia la institución educativa

Palabras Claves: calidad del servicio; educación superior; calidad percibida; ventaja competitiva

Abstract: The purpose of the following article is to propose a model that will enable us to analyze perception and effectiveness of the quality of service of fourth level program students in Ecuador. Currently, governmental agencies have enhanced their regulations to evaluate institutes which offer higher education. Methodology used for this experimentation, was mainly observation. We went through many articles of authors which focused on elaborating different kinds of models pointing to evaluation of quality of service of existing educational institutes, such as Serqual, Servperf and DIHEQS. The proposed model is "Perceived quality of post-grades". This model includes the following features: a) Teachers and teaching. b) Curricular contents. c) Technology and communications. d) Additional activities. and e) Organization and management, which are focused on loyalty and becomes a competitive advantage for the education institutes.

Key Words: quality of service; higher education; perceived quality; competitive advantage 


\section{Introducción}

La calidad en el servicio poco a poco toma gran importancia en todo tipo de negocios como, por ejemplo, la educación. Por esto los clientes exigen siempre lo mejor para cumplir sus necesidades y expectativas (Kotler, 2006). Anteriormente, la oferta era un poco más limitada, pero con la evolución que han tenido los mercados, han ocasionado que exista un aumento en la demanda, la calidad en el servicio empezó a tomar fuerza y a ser considerada un elemento básico para destacar y darle un valor agregado a las empresas (Kotler \& Armstrong, 2013).

El estado ecuatoriano crea universidades con alta calidad académica, en función del nivel educativo de los docentes y la administración (CEEACES, 2016). Uno de los procesos que requiere el Consejo de Educación Superior (CES) para evaluar la calidad universitaria es: servicios educativos de excelencia. (Consejo de Educaciòn Superior, 2013).

El problema es que el consumidor duda al momento de la compra debido a la gran elección de productos, lo que hace que la competencia haya alcanzado un alto nivel técnico para diferenciarse entre los demás, ocasionando que cliente sea muy exigente (Kotler \& Armstrong, 2013). Así, se demuestra que el servicio se ha vuelto fundamental en un negocio y es utilizado en las organizaciones como una herramienta de diferenciación esencial en términos de estrategia de marketing (Lovelock \& Wirtz, 2009).

El objetivo del presente trabajo es proponer un modelo para analizar la percepción y eficacia de la calidad de servicio de los estudiantes en programas de cuarto nivel en el Ecuador. Este aporte de información será útil y relevante para que en el futuro las universidades puedan evaluar la percepción de sus servicios (Rodriguez \& Munuera, 2012).

Al ser la calidad del servicio un elemento fundamental para la ventaja competitiva de las organizaciones, este trabajo aportará con ideas que los responsables de las instituciones educativas superiores deben considerar al ofrecer la educación de cuarto nivel. Es importante analizar las dimensiones de los modelos de los estudios publicados, conceptualizar la calidad del servicio en el sector de la Educación Superior, caracterizar los estudios de cuarto nivel en el Ecuador y determinar la metodología que se aplicará para luego crear una propuesta (Lovelock \& Wirtz, 2009).

\section{Revisión de la literatura}

\section{Calidad del servicio}

La calidad de servicio puede ser conceptualizada desde diferentes miradas o situaciones, es decir, que presenta una heterogeneidad de aportes. Debe estar fundamentada en las percepciones que los clientes tienen del servicio. Por lo tanto, la calidad del servicio es lo que todos clientes perciben al momento de adquirir un servicio (Joseph \& Joseph, 1997). Allí radica la relatividad de este concepto. Si el servicio iguala o supera la expectativa inicial será considerado un buen servicio, y creará una situación de lealtad del cliente, mientras que será catalogado de baja calidad cuando el cliente no esté satisfecho con la prestación (Camisón, Cruz, \& González, 2006). Según Lehtinen \& Lehtine (1982) "La calidad de un bien o servicio está compuesta por todos los atributos o propiedades que lo conforman y que le otorgan valor. La 
calidad de los productos se puede conocer con facilidad, pero no así la calidad de los servicios debido a sus características de intangibilidad, inseparabilidad, y caducidad." La interacción entre un cliente y los elementos de la organización que prestan el servicio es fundamental ya que la calidad dentro de los servicios se condiciona a la calidad percibida, y es relativa, esto se entiende como "el juicio del consumidor sobre la excelencia y superioridad de un producto" (Zeithaml, , Parasuraman, \& Berry, 1992). (Juran, 1996) destaca la puntualidad y el trato dado a los clientes como aspectos importantes de la calidad de servicio. Por ello dentro de la definición de calidad los ingredientes usuales para los servicios son: características, comportamiento, competitividad, rapidez, cortesía, capacidad de proceso, ausencia de errores, conformidad con estándares y procedimientos (Juran, 1996). La calidad objetiva es una perspectiva interna de la calidad con un enfoque centrado en la producción que busca eficiencia; y la calidad subjetiva es una visión externa que se logra con el cumplimiento de las necesidades, deseos y expectativas de los clientes (Vázquez, Rodríguez, \& Díaz, 1996).

La calidad del servicio depende fuertemente al momento de la interacción entre comprador y vendedor durante el encuentro de servicio (Zeithaml,, Parasuraman, \& Berry, 1992). En el marketing de productos, la calidad del producto suele depender de los atributos, beneficios y los valores agregados que ofrece, pero en el marketing de servicios, la calidad del servicio depende tanto de quien provee el servicio como de la calidad de la entrega (Kotler \& Armstrong, 2013).

Una empresa de servicio puede diferenciarse de las demás porque puede ofrecer una calidad de manera consistente mucho más alta que la que ofrecen sus competidores (Kotler, 2006). Igual que los fabricantes antes de ellos, la mayoría de las industrias de servicio ahora se han unido a la tendencia de calidad orientado al cliente, e igual que los mercadólogos de productos, los proveedores de servicios que debe identificar lo que esperan los clientes meta en cuanto a calidad de servicio (Kotler \& Armstrong, 2013).

\section{Componentes de la calidad del servicio}

Los clientes miden de diferentes formas la calidad de un servicio entre las que podemos mencionar las siguientes (Grande Esteban, 2014):

- Confiabilidad: es el grado de confianza para brindar un servicio a los clientes de manera más segura, precisa y estable (Colunga, 1995). La confiabilidad en ofrecer un buen servicio desde la primera vez y así con el tiempo ir formando un lazo estable entre el cliente y el proveedor (Grande Esteban, 2014).

- Accesibilidad: Las empresas de diferentes sectores que se dedican a ofrecer servicios deben brindarles a sus clientes las facilidades necesarias para que estos se comuniquen de manera rápida y eficaz con ellas y así poder recibir un servicio de calidad. Tanto así para poder dar un servicio más rápido las empresas utilizan la tecnología para ser más accesibles (Grande Esteban, 2014; Colunga, 1995).

- Respuesta: Es la disposición que tiene uno para atender y dar un servicio rápido (Colunga, 1995). Los consumidores cada vez son más exigentes en éste sentido y por la situación en 
la que están quieren que se les atienda rápido sin tener que esperar ya que el tiempo para ellos es muy valioso (Grande Esteban, 2014).

- Empatía: Esto quiere decir ponerse en los zapatos de los clientes, en su lugar para saber cómo se siente (Colunga, 1995). Darle la importancia que el cliente se merece, ocupar el lugar del cliente, valorar su tiempo y conocer cuáles son sus necesidades y exigencias (Grande Esteban, 2014).

- Seguridad: A los consumidores se les garantiza que los servicios que se están ofreciendo no carecen de riesgos, es importante que no existan dudas sobre los beneficios que se brindan (Grande Esteban, 2014; Colunga, 1995).

- Tangibles: Es todo lo que se puede ver como ejemplo las instalaciones físicas y el equipo de la organización deben transmitir una buena imagen y demostrar que son los mejores (Grande Esteban, 2014; Colunga, 1995).

\section{Calidad en educación superior}

La calidad de servicio en la educación superior ha evolucionado con el pasar del tiempo en todos los ámbitos tanto tecnológicos, sociales y financieros, estos cambios se han dado no solo a nivel local, sino también, mundial. Prueba de estos son los resultados de cambios internos y externos que han afectado aproximadamente a los últimos 40 años del siglo pasado (Aguilar V., 2005). Hasta los comienzos de la década de los 60’s se relacionaba la calidad de las instituciones educativas superiores con la tradición de la institución, estudiantes, docentes exclusivos y materiales; en la presente década, se la relaciona con el conocimiento impartido, conectado con el entorno y el impacto positivo que genera (Aguilar V., 2005)

La calidad en la educación superior tuvo una evolución completamente en la década de los 90’s y se cataloga como la que mejor satisface las necesidades individuales de los estudiantes y la sociedad, en la que la información proporciona a los usuarios las competencias básicas a nivel intelectual, moral físico, espirituales y afectivo con lo cual se pueda desempeñar en diversos ámbitos del entorno y la sociedad (Valdés \& Pérez, 1999).

A inicios del siglo XX la satisfacción de los estudiantes se relaciona con investigaciones que muestran que dicha satisfacción tiene un impacto positivo sobre la motivación, índices de retención y el rendimiento estudiantil (Elliot \& Shin, 2002); Los resultados de una investigación realizada a diferentes instituciones con la finalidad de conocer la valoración en la calidad de los modelos de las IES se obtuvo como resultado que la satisfacción estudiantil no es el componente más importante para determinar la calidad (Gento, 2002).

Existe diferentes modelos que usualmente se utilizan para la medición de la calidad en Educación Superior, El modelo Servperf ha sido utilizado por una escala más sencilla, convirtiendo a la percepción como el único factor para la evaluación de la calidad. (Duque \& Chaparro, 2012). Servqual se ha sido utilizado por una escala compuesto esto quiere decir que se utiliza diferentes ítems múltiples y su finalidad es medir las percepciones de los clientes con respecto a la calidad de cliente que percibe (Martínez Arguellos, Blanco, \& Castán, 2013). 
Serqualing que realiza estudios exploratorios involucrando variables de herramientas de evaluación generando un proceso de autoevaluación de la universidad y eso incluye al personal, docentes y los estudiantes (Aguilar, Maldonado, Massa, Ramírez, \& Rubio, 2005).

Adicionalmente se presentan los siguientes modelos de medición: Hiedqual el cual fue basado en SQ, pero aplicados a un contexto específico (Annamdevula \& Shekhar, 2012) y Hedpere creado por Firdaus Abdullah presentando altos niveles de confiabilidad (Ahmed \& Mehedi, 2014).

Debido a la gran cantidad de universidades que hay en el país se origina una mayor competencia, por lo que es importante encontrar y generar nuevos componentes que motiven la lealtad y permanencia de los estudiantes en las instituciones de educación superior, esto dependerá básicamente del instrumento que se seleccione para identificar los aspectos importantes desde la perspectiva de los estudiantes y con ello las acciones que deberá tomar la institución para cumplirlas (Duque \& Chaparro, 2012).

\section{El concepto de calidad educativa en el Ecuador}

Es cierto que la educación no puede entenderse como un producto físico o elaborado sino como un servicio que se presta a los alumnos (Sylva Charvet, 2014). Pero, al igual que ocurre con otros servicios, la naturaleza de este servicio resulta difícil de describir, así como los métodos para evaluar la calidad (Seneca, 2015).

Para agregar en la Constitución política de nuestro del ecuador establece

- "Art. 26.- La educación es un derecho de las personas a lo largo de su vida y un deber ineludible e inexcusable del Estado. Constituye un área prioritaria de la política pública y de la inversión estatal, garantía de la igualdad e inclusión social y condición indispensable para el buen vivir. Las personas, las familias y la sociedad tienen el derecho y la responsabilidad de participar en el proceso educativo" (Constitución de la República del Ecuador, 2008, pág. 16).

- “Art. 27.- La educación se centrará en el ser humano y garantizará su desarrollo holístico, en el marco del respeto a los derechos humanos, al medio ambiente sustentable y a la democracia; será participativa, obligatoria, intercultural, democrática, incluyente y diversa, de calidad y calidez; impulsará la equidad de género, la justicia, la solidaridad y la paz; estimulará el sentido crítico, el arte y la cultura física, la iniciativa individual y comunitaria, y el desarrollo de competencias y capacidades para crear y trabajar" (Constitución de la República del Ecuador, 2008, pág. 16).

- “Art. 173.- Evaluación Interna, Externa, Acreditación, Categorización y Aseguramiento de la Calidad. - El Consejo de Evaluación, Acreditación y Aseguramiento de la Calidad de la Educación Superior normará la autoevaluación institucional, y ejecutará los procesos de evaluación externa, acreditación, clasificación académica y el aseguramiento de la calidad" (CEEACES, 2016, pág. 120). 
Las universidades, escuelas politécnicas, institutos superiores técnicos, tecnológicos, pedagógicos, de artes y conservatorios superiores del país, tanto públicos, como particulares, sus carreras y programas, deberán someterse en forma obligatoria a la evaluación interna y externa, a la acreditación, a la clasificación académica y al aseguramiento de la calidad (CEEACES, 2016, pág. 121).

- Art. 174.- Funciones del Consejo de Evaluación, Acreditación y Aseguramiento de la Calidad de la Educación Superior. - Son funciones del Consejo de Evaluación, Acreditación y Aseguramiento de la Calidad de la Educación Superior Otorgar certificados de acreditación institucional, así como para programas y carreras, a las instituciones de educación superior y unidades académicas que hayan cumplido con todos los requisitos exigidos para el efecto. Este certificado de acreditación tendrá una vigencia de cinco años y no podrá estar condicionado (CEEACES, 2016, pág. 121).

\section{Tipos de estándares para la calidad en la educación}

Según El Ministerio de Educación (2012), ha diseñado 4 tipos de estándares con el objetivo de asegurar que los estudiantes obtengan una educación de calidad:

- Estándares de Gestión Escolar: Hacen referencia a procesos de gestión y a prácticas institucionales que contribuyen a la formación de los estudiantes. Además, aportan en el desarrollo profesional de los docentes de la institución educativa y permiten que ésta se aproxime a su funcionamiento ideal (Ministerio de Educación, 2012).

- Estándares de Desempeño Profesional: Son las normas que debe seguir un docente competente; es decir, las prácticas que tienen una mayor relación positiva con la formación que se desea que los estudiantes alcancen (Ministerio de Educación, 2012).

- Estándares de Aprendizaje: Son descripciones de los logros de aprendizaje que los estudiantes deben alcanzar a lo largo de la trayectoria escolar: desde la Educación Inicial hasta el Bachillerato (Ministerio de Educación, 2012).

- Estándares de infraestructura: Constituyen los requisitos esenciales, orientados a determinar las particularidades que los espacios y ambientes escolares que deben tener para contribuir al alcance de resultados deseados en la formación de estudiantes y en la efectividad de la labor docente (Ministerio de Educación, 2012).

\section{Educación superior de cuarto nivel o postgrado en el Ecuador}

Hoy en día vivimos en un ambiente laboral sumamente competitivo, que solo teniendo un título de tercer nivel no basta para poder desarrollarse plenamente en su vida profesional, motivo por el cual algunos profesionales continua con su preparación académica, para ello muchas universidades en el Ecuador ven una oportunidad de generar ingresos y por ese motivo crean carreras de postgrado a fin de satisfacer las necesidades, deseos y expectativas de los profesionales del país; y, así mismo contribuyen en el desarrollo social, humano, económico y tecnológico de nuestro país (CONEA, 2014). 
El objetivo fundamental para realizar una carrera de postgrado es la de ofrecer a diferentes profesionales la oportunidad de ir más del simple manejo de los conocimientos adquiridos en su respectiva profesión, para entrar en el mundo de la investigación, practica y la producción de conocimientos de calidad, con la finalidad de obtener profesionales con características que lo oferten al mercado de una manera mucho más competitiva (CONEA, 2014).

Según La Ley Orgánica de Educación Superior -LOES- que está vigente en el país desde el 12 de octubre del 2010 el cuarto nivel o postgrado, está orientado al entrenamiento profesional y académico avanzado o a la especialización científica y de investigación en un área específica (Sylva Charvet, Cultura de género y calidad, 2016). También lo podemos definir con el grado académico que busca ampliar, desarrollar, conocer y profundizar en una área específica o disciplina del conocimiento. Concede a la persona de las herramientas que la habilitan para profundizar teórica e instrumentalmente en un campo al que se quiere formar (Sylva Charvet, 2016).

Cada maestría debe estar orientada al desarrollo de un área de la correspondiente carrera con fines académicos o profesionales, y están enfocadas a la formación en el conocimiento y aplicación de métodos de investigación que tengan una capacidad innovadora, técnica y metodológica para la solución de problemas en el ejercicio profesional. (Consejo de Educaciòn Superior, 2013) El estudiante deberá realizar entre 64 y 96 créditos, con una duración mínima de dos semestres, para obtener el grado académico de maestría profesional, con dedicación a tiempo completo. Para obtener el grado académico de maestría en investigación se deberá completar entre 96 y 120 créditos, con una duración mínima de tres semestres, con dedicación a tiempo completo (Consejo de Educaciòn Superior, 2013).

En este año 2017 el Senescyt ha registrado 31 universidades que ofrecen diferentes carreras de postgrados. Los profesionales pueden acceder a esta carrera, su principal requisito es que tenga un título de tercer nivel. En el anexo 1 podrá visualizar las universidades que ofrezcan postgrado (Senescyt, 2017). A continuación, se demostraras algunos artículos del Consejo de Educación Superior del Ecuador que son necesarios para que un programa de "maestría sea aprobado.

Artículo 22.- Unidades de organización curricular en los programas de posgrado. - Estas unidades son" (Consejo de Educación Superior, 2012, pág. 14):

“1. Unidad básica. - Será incluida en aquellos programas con metodologías multi, inter o trans disciplinarios. Establece las bases teóricas y metodológicas de la referida organización del conocimiento" (Consejo de Educación Superior, 2012, pág. 14);

"2. Unidad disciplinar, multi disciplinar y/o inter disciplinar avanzada.- Contiene los fundamentos teóricos, epistemológicos y metodológicos de la o las disciplinas y campos formativos que conforman el programa académico" (Consejo de Educación Superior, 2012, pág. $14)$

“3. Unidad de titulación. - Está orientada a la fundamentación teórica-metodológica y a la generación de una adecuada base empírica, que garantice un trabajo de titulación que contribuya 
al desarrollo de las profesiones, los saberes, la tecnología o las artes, y las ciencias. Los trabajos de titulación deberán se $\mathrm{r}$ individuales" (Consejo de Educación Superior, 2012, pág. 14)”; "Artículo 29.- Campos de formación de la educación superior de posgrado o de cuarto nivel. En este nivel los campos de formación se organizarán de la siguiente manera" (Consejo de Educación Superior, 2012, pág. 17).:

"1. Formación profesional avanzada.- Comprende la profundización e integración del conocimiento metodológico y tecnológico de un campo científico y/o profesional específico" (Consejo de Educación Superior, 2012, pág. 17).

“2. Investigación avanzada. - Comprende el desarrollo de la investigación básica o aplicada, vinculadas a las líneas de investigación del programa, utilizando métodos de carácter disciplinar, multi, inter o trans disciplinar, según sea el caso. En este campo formativo se incluirá el trabajo de titulación" (Consejo de Educación Superior, 2012, pág. 17).

“3. Formación epistemológica. - Supone la integración de diversas perspectivas epistemológicas. teóricas y culturales en ámbitos inter y/o trans disciplinarios, a fin de lograr la integralidad de la formación del estudiante. Este campo deberá estar articulado con el campo de investigación avanzada" (Consejo de Educación Superior, 2012, pág. 17).

"Artículo 64.- Denominación de los títulos. - Las instituciones de educación superior sólo podrán expedir los siguientes títulos, conforme a los distintos niveles y tipos de carreras de la educación superior" (Consejo de Educación Superior, 2012, pág. 28).

En la educación superior de posgrado: a. Especialista en el correspondiente ámbito profesional. b. Especialista médico o especialista odontólogo en el correspondiente ámbito académico o profesional. Este título habilitará para la carrera de profesor e investigador auxiliar y agregado en la educación superior. c. Magíster profesional en el correspondiente ámbito profesional. d. Magíster en investigación en el correspondiente ámbito académico. e. Doctor (equivalente a $\mathrm{PhD}$ ) en el correspondiente ámbito académico (Consejo de Educación Superior, 2012, pág. 40).

\section{Metodología}

La metodología que se aplicará en cualitativa, mediante la observación de documentos para luego crear la propuesta de un instrumento que se pueda aplicar en el contexto ecuatoriano. A continuación, se hace referencia de 3 modelos que son herramientas diseñadas para la medición de la calidad del servicio y son Serqual, servperf y DIHEQS (Cronin \& Taylor, 1994; Martínez Arguellos, Blanco, \& Castán, 2013).

Servqual

Debido a la necesidad de la satisfacción del cliente para las empresas e instituciones en general, nacieron herramientas para medir la calidad en el servicio y así conocer el nivel de satisfacción de los mismos; tal como la herramienta SERVQUAL; misma que fue desarrollada por (Zeithaml,, Parasuraman, \& Berry, 1992) donde se evalúan los factores claves para determinar dimensiones del servicio

1) Tangibilidad: las instalaciones físicas, equipos de oficina, y el aspecto del personal, todo lo que se puede visualizar y palpar. (Zeithaml, , Parasuraman, \& Berry, 1992)

2) Fiabilidad: práctica para realizar el servicio prometido en forma confiable y precisa. (Zeithaml, , Parasuraman, \& Berry, 1992) 
3) Capacidad de respuesta: habilidad para ayudar a los clientes y proporcionar rápido servicio (Parasuraman, Zeithaml, \& Berry, 1988).

4) Seguridad: el conocimiento que tienen los empleados y su capacidad para inspirar confianza al cliente al momento de ofrecer un producto. (Parasuraman, Zeithaml, \& Berry, 1988)

5) Empatía: el cuidado, la atención individualizada que la empresa proporciona a sus clientes (Parasuraman, Zeithaml, \& Berry, 1988)

En la figura 1 se muestra el modelo de evaluación del cliente sobre la calidad del servicio en que se basa la metodología Servqual.

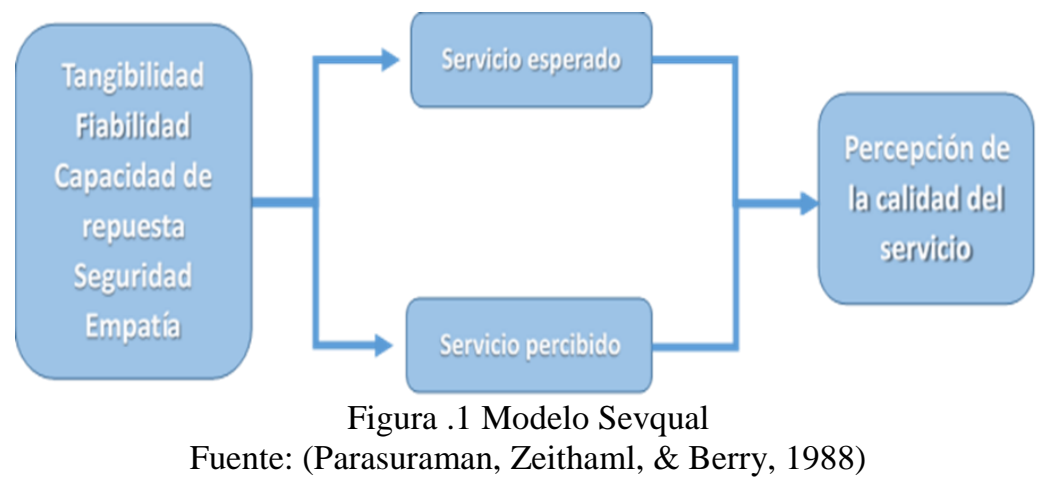

\section{Servperf}

El modelo Servperf utiliza exclusivamente la percepción como una buena aproximación a la satisfacción del cliente, definida ésta como "el nivel del estado de ánimo de una persona que resulta de comparar el rendimiento percibido de un producto o servicio con sus expectativas" (Kotler, 2006). Otros autores mencionan que el modelo SERVPERF (Cronin \& Taylor, 1994) utiliza exclusivamente la percepción como mejor aproximación a la satisfacción. En la metodología empleada en el modelo SERVPEF se únicamente hay una encuesta (relacionada con la percepción del servicio y no con las expectativas) y el trabajo de interpretación y análisis de resultados es más sencillo además este modelo presenta datos más reales en la percepción del servicio prestado; además los autores concluyeron que la satisfacción del cliente es producto de la calidad del servicio y que ésta influye en la intención de compra del cliente. También concluyeron que la calidad del servicio influye más en la satisfacción del cliente que en la intención de compra (Cronin \& Taylor, 1992)

\section{El modelo "Distance Higher Education Quality Service” (DIHEQS)}

El modelo DIHEQS cuenta con las dimensiones consideradas en escalas que miden la calidad de servicio en educación superior tradicional, así como la escasa literatura existente sobre la educación a distancia. Con el uso de este modelo se basa la incorporación de las dimensiones que son consideradas con mayor frecuencia por los investigadores (Araya-Castillo \& Bernardo, 2013). El modelo "Distance Higher Education Quality Service” (DIHEQS), cuenta con las siguientes dimensiones (Martínez-Argüelles, Castán, \& Juan, 2010) La dimensión "profesores y enseñanza" cuenta con los siguientes aspectos relacionados con el carácter, actitud, experiencia, conocimiento, comunicación grupal o personal tiempo de repuesta y motivación al estudiante 
(Holdford \& Patkar, 2003) (Bhat \& Bhat, 2012). La dimensión "evaluación y feedback" cuenta con los siguientes aspectos relacionados con el tiempo de entrega de los resultados, nivel de dificultad, feedback y relación con los contenidos del curso (Entwistle \& Tait, 1990; Tan \& Kek, 2004).

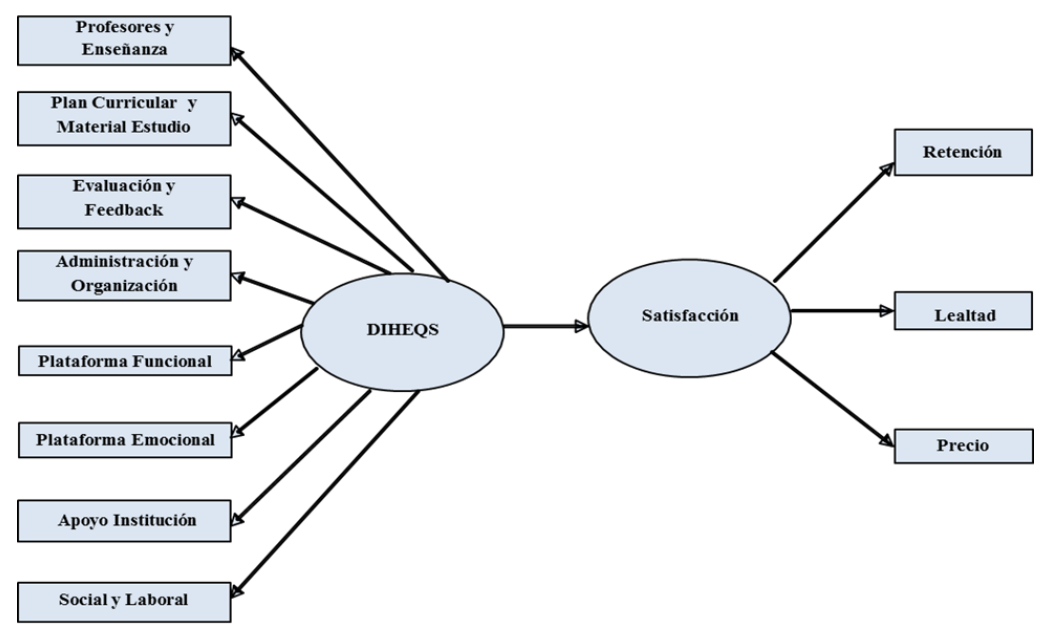

Figura .2 Modelo Distance Higher Education Quality Service (DIHEQS), Fuente: (Martínez-Argüelles, Castán, \& Juan, 2010)

La dimensión "material de estudio" se relaciona el contenido, conocimientos actuales, base de información y que tenga relación con el ámbito profesional dirigido la carrera que se está enfocando (Entwistle \& Tait, 1990) (Martínez-Argüelles, Castán, \& Juan, 2010). La dimensión "plan curricular" cuenta con los siguientes aspectos relacionados con la estructura del plan de estudios, contenido de cada materia y la duración (Munteanu, Ceobanu, Bobalca, \& Anto, 2010).

La dimensión "administración y organización” incluye aspectos relacionados con el nivel de comunicación, planificación de las actividades, gestión de requerimientos, asesoramiento de proceso en los procesos además el respeto y el servicio de los directores y/o coordinadores hacia los estudiantes (Tan \& Kek, 2004) (Holdford \& Patkar, 2003). La dimensión "plataforma virtual" incluye aspectos relacionados al uso de la plataforma, la estética, contenido, conexión y portabilidad vinculación con otros sistemas (Martínez-Argüelles, Castán, \& Juan, 2010). La dimensión "apoyo institución" considera aspectos relacionados con las alianzas o convenio que cuenta la institución, el asesoramiento que reciben los estudiantes, las facilidades Y diferentes Forma de pago y con otros estudiantes del programa (Joseph \& Joseph, 1997; Kwan \& Ng, 1999).

\section{La propuesta}

La propuesta es crear un modelo para analizar la percepción de la calidad de servicio en la educación superior, tomando las cuentas las dimensione y los factores que se requiera, pero la forma de calificar será completamente diferente.

El fin de esta investigación es plantear un modelo adecuado que cumpla con todos los estándares para medir la percepción de la calidad de servicio en las instituciones educativa de 
cuarto nivel. Por este motivo se escogerá diferentes dimensiones consideradas en escalas que miden la calidad. Esta propuesta tiene como finalidad utilizar dimensiones que son consideradas con mayor frecuencia por los investigadores y una dimensión que va de la mano con las tendencias del mercado la cual se debe tener en cuenta al momento incluirla. Por esta razón, se propone que el modelo "Calidad percibida en posgrado" (CPEP), contienen las siguientes dimensiones: a) Profesores y enseñanza; b) Malla curricular; c) Tecnología y comunicación; d) Actividades adicionales; y d) Administración y organización. En la tabla 1 se visualiza la validación de contenido del modelo propuesto.

Tabla 1: Validación de contenido de las dimensiones propuestas en el modelo (CPEP)

\begin{tabular}{cc}
\hline Dimensiones & Escalas previas en educación superior \\
\hline Profesores y & Entwistle y Tait (1990); Harvey (1995); Hill (1995); Shank et al. (1995); Tan y \\
enseñanza & Kek (2004); Marzo-Navarro et al. (2005); Douglas et al. (2008); Martínez- \\
& Argüelles et al. (2010) \\
Malla curricular & Entwistle y Tait (1990); Martínez-Argüielles et al. (2010); Munteanu et al. \\
& (2010); Torres y Araya-Castillo (2010); Kimani et al. (2011); Jain et al. (2013). \\
Tecnología y & (Pardo Gómez, Izquierdo Lao, \& Fuentes González, 2005); (Saez Lopez \& \\
comunicación & Ruiz Ruiz, 2012); (Alva Arce, 2011) \\
Apoyo institucional & Tan y Kek (2004); Gruber et al. (2010); Martínez-Argüelles et al. (2010); \\
& Harvey (1995); Hill (1995); Joseph y Joseph (1997); Hampton (1993) \\
Administración y & Harvey (1995); Morales y Calderón (1999); Holdford y Patkar (2003); Gruber \\
organización & et al. (2010); Martínez-Argüelles et al. (2010); Torres y Araya-Castillo (2010); \\
& Kimani et al. (2011); Sultan y Wong (2011). \\
\hline
\end{tabular}
Fuente: Elaboración propia

La dimensión del profesor y enseñanza se componen en: experiencia, imagen, responde claridad, puntualidad, atento a las necesidades del estudiante, organizado y profesionalismo Malla curricular aspectos actuales del mercado, relacionado al mercado laboral y la duración

Tecnología y comunicación se compone en: medios de comunicación, conocimiento de herramientas tecnológicas, y calidad y funcionalidad de los equipos. Apoyo institucional se compone en: Alianza con otras instituciones, bolsa de trabajo, pasantías institucionales y olimpiadas. Administración y organización se compone en: requerimiento solicitado, envió de información, trato, instalaciones, y respeto con el personal.

Dado esto, se propone diseñar una herramienta que permita medir la calidad de servicio entregada por las universidades que ofrecen programas de cuarto nivel. Se señala que la educación presenta dinámicas similares. Es así como altos niveles de competencia en los sectores de educación superior se observan (Malhotra \& Birks, 2008). El diferenciador de esta herramienta es la calificación, en la cual se pedirá que se califique desde un rango de $1 \%$ a $100 \%$, pero también está la opción para calificar más del 100\%, de esta forma se puede reflejar que no solo cumplió las expectativas del cliente si no que las superó. Para una institución educativa es importante agregar esta condición, para que así sepan cuáles son los factores que no solo cumplieron las expectativas, sino que la superaron y esto puede generar una ventaja competitiva hacia la demás, en la cual no solo se debe cumplir las perceptivas del estudiante sino superarla. Según (Porter, 1985) "La ventaja competitiva crece principalmente en razón del valor de una empresa que es capaz de generar. El concepto de valor representa lo que los compradores están dispuestos a pagar, y el crecimiento de este valor a un nivel superior se debe a la capacidad 
para ofrecer precios más bajos en relación a los competidores por beneficios semejantes, o proporcionar beneficios únicos en el mercado que puedan compensar los precios más elevados.

Una empresa se considera rentable si el valor que es capaz de generar es más elevado de los costos generados por la creación del producto. A nivel general, podemos afirmar que la finalidad de cualquier estrategia de empresa es generar un valor adjunto para los compradores, que sea más elevado del costo empleado para generar el producto. Por lo cual en lugar de los costos deberíamos utilizar el concepto de valor en el análisis de la posición competitiva (Kotler \& Armstrong, 2013; Porter, 1985).

Las ventajas de este modelo son: Conocer los factores más relevantes que el cliente o estudiante percibe, Conocer cuáles son los factores que necesitan realizar algunas mejoras, así se podrá implementar plan de mejorar los factores que superan las expectativas que se convierten en una ventaja competitiva y los factores más relevantes como una retroalimentación a los que necesitan mejorar (Kotler \& Armstrong, 2013). Observar el modelo en la figura 3 e instrumento en el anexo 1

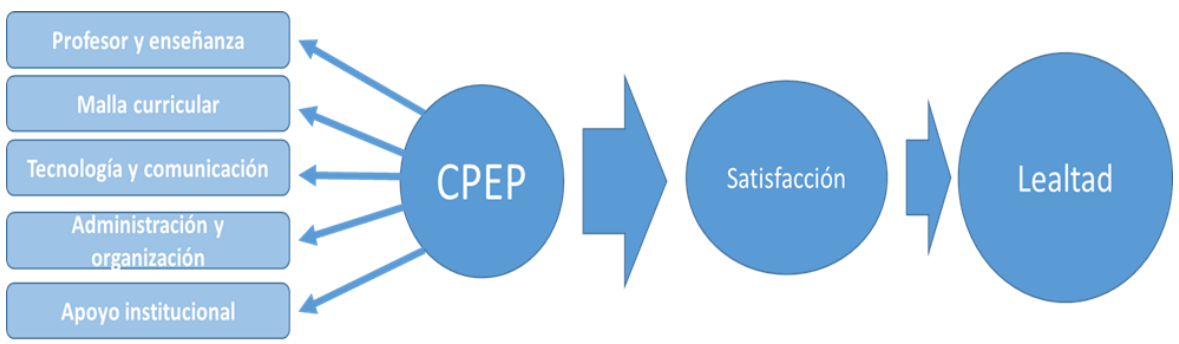

Figura. 3 Modelo "Calidad percibida en posgrado" (CPEP), Fuente: Elaboración propia

\section{Conclusiones}

Las instituciones gubernamentales del Ecuador realizan constantemente evaluaciones y crean procedimientos para calificar a las instituciones de educación superior, esto significa que si no mantienen o regulan sus estándares calidad se verá afectada su calificación (CEEACES, 2016). Existen un gran número universidades en el Ecuador por lo tanto los estudiantes tienen la facilidad de elegir y exigir el servicio educativo más idóneo de acuerdo sus necesidades que cumplan sus expectativas (CEEACES, 2016).

Cada día los clientes son más exigentes al momento de adquirir un producto o servicio, por lo tanto, las instituciones educativas se ven en la necesidad de implementar nuevos planes estratégicos para atraer e incrementar su número de clientes (Joseph \& Joseph, 1997). Las dimensiones para evaluar la perspectiva de la calidad han evolucionado significativamente y es evidente que no solo miden lo tangible sino también intangible, además las instituciones educativas a nivel superior que ofrecen las modalidades presenciales y a distancia deben contar con los recursos y equipos necesarios para estar actualizados con el mercado actual (Colunga, 1995).

La tecnología y la comunicación son necesario ser evaluar por las siguientes razones: si estas en contacto con tu cliente frecuentemente puedes conocer más sus necesidades y el uso de 
equipo tecnológicos apropiados sirve para optimizar el tiempo del estudiante y que sean usable (Pardo Gómez, Izquierdo Lao, \& Fuentes González, 2005). La metodología que se usó para formar este modelo estuvo fundada por varios autores que realizaron diferentes estudios académicos basados en el servicio de la educación superior (Malhotra \& Birks, 2008). Por medio del uso del método de escala en este instrumento podemos medir la perspectiva de los estudiantes hacia las instituciones educativas, y de esta forma conocer las fortalezas y debilidades que se presenten para luego implementar un plan de mejoras (Duque \& Chaparro, 2012).

Un riesgo que puede surgir al momento de aplicar este instrumento es que los estudiantes no respondan adecuadamente por este motivo se debe realizar un plan estratégico para que incentive a contestar la encuesta de forma adecuada y honesta.

\section{Bibliografía}

Aguilar, R., Maldonado, J., Massa, P., Ramírez, L., \& Rubio, M. (2005). Proceso de autoevaluación de los programas de educación a distancia basado en el proyecto. Loja: UTPL.

Aguilar, V. (2005). El concepto de calidad en la educación universitaria: Clave para el logro de la competitividad institucional. Revista Iberoamericana de Educación.

Ahmed, S., \& Mehedi, M. (2014). Measuring service quality of a higher educational institute towards student satisfaction. American Journal of Educational Research, 447-455.

Alva Arce, R. (2011). Las Tecnologías de información y comunicación como Las Tecnologías de información y comunicación como de educación con mención en docencia en el nivel. Obtenido de http://sisbib.unmsm.edu.pe: http://sisbib.unmsm.edu.pe/bibVirtualData/Tesis\%20para\%20marcaci\%C3\%B3n3\%20\% 28para\%20Inform\%C3\%A1tica\%29/2011/alva_ar/alvar_ar.pdf

Annamdevula, S., \& Shekhar, R. (2012). Development of HiEdQUAL for measuring service quality in Indian higher education sector. International Journal of Innovation, Management and Technology, 412-416.

Araya-Castillo, L., \& Bernardo, M. (Diciembre de 2013). https://www.academia.edu. Obtenido de https://www.academia.edu/8167491/DTUM-17-LAraya: https://www.academia.edu/8167491/DTUM-17-LAraya

Bhat, R., \& Bhat, K. (2012). Service Quality in Management, Education in Gujarat State: A Study of. Perception and Preferences. e Reflection, 1, 244-256.

Camisón, C., Cruz, S., \& González, T. (2006). Gestión De La Calidad: Conceptos, Enfoques, Modelos Y Sistemas. Madrid: Pearson Educación, S. A.

CEEACES. (2016). Obtenido de http://www.ceaaces.gob.ec/sitio/acreditacion-y-categorizacion/ 
Colunga, D. (1995). La calidad de servicio. Mexico: Panorama.

CONEA. (2014). www.issuu.com. Obtenido de file://Users/GMM/Documents/Maestria\%20uees/Metodologia\%20investigacion\%201/M andato+14.Pdf

Consejo de Educación Superior. (2012). http://www.snna.gob.e. Obtenido de http://www.snna.gob.ec/wp-content/themes/institucion/dwpages/Descargas/regimen_academico.pdf

Consejo de Educaciòn Superior. (2013). Reglamento de regimen academico. Quito.

Constitución de la República del Ecuador. (2008). Obtenido de https://www.google.com.ec/url?sa=t\&rct=j\&q=\&esrc=s\&source=web\&cd=3\&cad=rja\&u act=8\&ved=0ahUKEwjCiLK46aTTAhWDPCYKHXWVBEMQFggtMAI\&url=http\%3A $\% 2 \mathrm{~F} \% 2 \mathrm{Fwww}$.turismo.gob.ec\%2Fwpcontent $\% 2$ Fuploads\%2Fdownloads $\% 2$ F2014\%2F03\%2FconstitucionMINTUR.docx\&usg=AFQjCNEPXE--7G

Cronin, J., \& Taylor, s. (1994). servperf versus servQual: reconciling performancebased and perceptions minus expectations measurement of service quality”, in: Journal of Marketing. Journal of Marketing.

Cronin, J., \& Taylor, s. (1992). Measuring service quality: a reexamination and extension. Journal of Marketing.

Duque, J., \& Chaparro, C. (2012). Medición de la percepción de la calidad del servicio de educación por parte de los estudiantes de la UPTC Duitama. Criterio Libre, 159-192.

Elliot, K., \& Shin, D. (2002). Student Satisfaction: an alternative approach to assessing this important concept. Journal of Higher Policy and management, 197-209.

Entwistle, N., \& Tait, H. (1990). Approaches to Learning, Evaluation of Teaching, and Preferences for Contrasting Academic Environments. Higher Education, 19(2), 169-194.

Gento, S. (2002). La evaluación de la satisfacción educativa en un enfoque de calidad institucional. Madrid: Prentice Hall.

Grande Esteban, I. (2014). Marketing De Los Servicios. ESIC.

Hampton, G. (1993). Gap Analysis of College Student Satisfaction as a Measure of Professional Service Quality. Journal of Professional Services Marketing, Vol. 9, nº 1, 115-128.

Harvey, L. (1995). Quality in Higher Education Vol. 1, nº 1. The Quality Agenda. 
Hernández Sampieri, R., Fernández Coll, C., \& Baptista Lucio, P. (2014). Metodología de la Investigación. México D.F.: Mc. Graw Hill.

Hill, F.-M. (1995). Managing Services Quality in Higher Education: The Role of the Students as Primary Consumers. En Q. A. Education. Vol.3, no 3.

Holdford, D., \& Patkar, A. (2003). Identification of the Service Quality Dimensions of Pharmaceutical Education. American Journal of Pharmaceutical Education, 67(4), 1-11.

IBM. (2015). IBM SPSS Statistics 22 Core System Guía del usuario.

Jain, S., \& Gupta, G. (2004). Measuring Service Quality: SERVQUAL vs. SERVPERF Scale. VIKALPA Vol. 29, nº 2, 25-37.

Joseph, M., \& Joseph, B. (1997). Service Quality en Education: A Student Perspective. Quality Assurance in Education, 5(1), 15-21.

Juran, J. (1996). Juran y la calidad por el diseño. illustrated.

KImani, S., Kagira, E., \& Kendi, L. (2011). Comparative Analysis of Business Students' Perceptions of Service. International Journal of Business Administration, Vol. 2, $\mathrm{n}^{\mathbf{0}}$ 1, 98 112.

Kinnear, T., \& Taylor, J. (1998). Investigación de Mercados. Bogotá: McGraw-Hill.

Kotler. (2006). Dirección de mercadotecnia. 8ª ed. México: Pearson-Prentice Hall.

Kotler, P., \& Armstrong, G. (2013). Fundamentos de marketing. Mexico: Pearson Educaciòn .

Kwan, Y., \& Ng, W. (1999). Quality Indicators in Higher Education-Comparing Hong Kong and China Students. Managerial Auditing Journal, 14(1-2), 20-27.

Lehtinen, J., \& Lehtinen, O. (1982). Service quality: A study pf quañity dimensions. Service Management Institute.

Lovelock, C., \& Wirtz, J. (2009). Marketing de Servicios. Mexico: Pearson Educación.

Malhotra, N., \& Birks, D. (2008). Marketing Research An Applied Approach,. England: Pearson Education Limited.

Martínez Arguellos, M., Blanco, M., \& Castán , J. (2013). Las dimensiones de la calidad del servicio percibida en entornor virtuales de formación superior. Revista de Universidad y Sociedad del Conocimiento, 89-106.

Martínez-Argüelles, M., Castán, J., \& Juan, A. (2010). How do Students Measure Service Quality in e- Learning? A Case Study Regarding an Internet-Based University. Electronic 
Journal of e-Learning, 8(2), 151-160.

Marzo-Navarro, M., Pedraja-Iglesias, M., \& Rivera-Torres, P. (2005). A new management element for universities: satisfaction with the offered courses. International Journal of Educational Management, 505- 526.

Ministerio de Educación. (2012). https://educacion.gob.ec. Obtenido de https://educacion.gob.ec/wp-content/uploads/downloads/2013/03/estandares_2012.pdf

Morales, M., \& Calderón, L. (1999). Assessing Service Quality in Schools of Business: Dimensions of Service Quality in Continuing Professional Education (CPE). Cuadernos de Difusión, ESAN, Vol. 5, 125-140.

Munteanu, C., Ceobanu, C., Bobalca, C., \& Anto, O. (2010). An analysis of customer satisfaction in a higher education context. International Journal of Public Sector Management, 23(2), 124-140.

Parasuraman, Zeithaml, \& Berry. (1988). "servQual: a multiple-item scale for measuring consumer perceptions of services quality", in: Journal of Retailing.

Pardo Gómez, M., Izquierdo Lao, J., \& Fuentes González, H. (2005). Comunicaciones en la dinámica del proceso docente educativo en la educación superior. Obtenido de CIVE 2005 Congreso Internacional Virtual de Educación: http://sedici.unlp.edu.ar/bitstream/handle/10915/24592/Documento_completo.pdf?sequen $\mathrm{ce}=1$

Pedret, R., Sagnier, L., \& Camp, F. (2000). Herramientas para Segmentar Mercados y Posicionar Productos. Bilbao: Ediciones Deusto.

Porter, M. (1985). Competitive Advantage: Creating and Sustaining Superior Performance. New York: Free Press.

Rodriguez, A., \& Munuera, J. (2012). Estrategias de Marketing Un enfoque basado en el proceso de direcciòn. Madrid: Grafica Denon.

Saez Lopez, J., \& Ruiz Ruiz, J. (Diciembre de 2012). Metodología Didáctica Y Tecnología Educativa En El Desarrollo De Las Competencias Cognitivas: Aplicación En Contextos Universitarios. Obtenido de http://www.ugr.es: http://www.ugr.es/ recfpro/rev163COL9.pdf

Seneca. (2015). ocw.usal.es. Obtenido de http://ocw.usal.es/ciencias-sociales-1/investigacionevaluativa-en-educacion/contenidos/Calidad.pdf

Senescyt. (2017). http://www.senescyt.gob.ec/. Obtenido de http://www.senescyt.gob.ec/posgradoecuador/index.html 
Sultan, P., \& Wong, H. (2011). Service Quality in a Higher Education Context: Antecedents and Dimension. International Review of Business Research Papers, Vol. 7, nº 2, 11-20.

Sylva Charvet, E. (2014). https://issuu.com. Obtenido de https://issuu.com/ceaacesec/docs/ceaaces-resg_2015_calidad_de_la_edu

Sylva Charvet, E. (2016). Cultura de género y calidad. IAEN.

Tan, K., \& Kek, S. (2004). Service Quality in Higher Education Using an Enhanced SERVQUAL Approach. Quality in Higher Education, 10(1), 17-24.

Torres, E., \& Araya Castillo, L. (2010). Construcción de una escala para medir la calidad del servicio de las universidades: Una Aplicación al Contexto Chileno. Revista de Ciencias Sociales, Universidad del Zulia, Vol. 16, $n^{\circ}$ 1, 54-67.

Valdés, H., \& Pérez, F. (1999). Calidad de la Educación Básica y su Evaluación. La Habana: Pueblo y Educación.

Vázquez, R., Rodríguez, I., \& Díaz, M. (1996). Estructura multidimensional de la calidad de servicio en cadenas de supermercados:. Madrid: universidad de oviedo - facultad de ciencias.

Zeithaml, , V., Parasuraman, ,., \& Berry, L. (1992). Calidad Total en la Gestión de Servicios. Ediciones Díaz de Santos.

\section{Anexos 1}

\section{Instrumento del modelo CPEP}

\begin{tabular}{cc}
\hline Rango de edad & Nombre del programa \\
\hline $24-30$ años & \\
$31-35$ años & Genero \\
$36-40$ años & Masculino \\
$41-45$ años & Femenino \\
Más de 46 años & \\
\hline
\end{tabular}

Califique las siguientes preguntas del 1 al 10, siendo 1 la calificación más baja y 10 la más alta.

\begin{tabular}{cc}
\hline$\#$ & Preguntas (Factores) \\
\hline & Profesores y enseñanza \\
\hline 2 & La presentación personal de los profesores con los estándares profesionales \\
\hline 3 & $\begin{array}{c}\text { Los profesores transmiten un nivel de conocimiento teórico y práctico actualizado. } \\
\text { curso que va a realizar. }\end{array}$ \\
\hline 4 & $\begin{array}{c}\text { Los profesores está pendiente y preocupado por el aprendizaje, necesidades, motivan por la } \\
\text { materia y fomentan la participación de los estudiantes. }\end{array}$ \\
\hline
\end{tabular}


5 Los profesores son atentos, educados y respetuosos al relacionarse con los estudiantes.

6 Los profesores usan método estratégico para transmitir el conocimiento teórico.

$\underline{\text { Tecnología y comunicación }}$

$7 \quad$ Calidad, cobertura y rapidez del wifi

8 Funcionamiento de los equipos tecnológicos (computadoras, infocus, etc)

9 Existe una comunicación fluida y de confianza por medio de un Smartphone

10 Existe una comunicación fluida y de confianza por medio de un teléfono convencional

11 Existe una comunicación fluida y de confianza por medio de un correo electrónico

12 Existe una comunicación fluida y de confianza por medio de un redes sociales

13 Existe una comunicación fluida y de confianza entre los directores de carrera y estudiantes.

14 Existe una comunicación fluida y de confianza entre el personal administrativo y estudiantes.

15 Existe una comunicación fluida y de confianza entre los profesores y estudiantes.

16 Conocimiento de herramientas tecnológicas para uso académico

\section{$\underline{\text { Malla curricular }}$}

17 Las notas son asignadas por los profesores siguiendo únicamente criterios de objetividad.

18 El contenido programático de las asignaturas propuesto por los profesores se lleva a cabo totalmente durante su tiempo en la maestría.

19 Los programas están actualizados a las necesidades del mercado actual.

\section{$\underline{\text { Administración y organización }}$}

20 Las instalaciones físicas son ordenas, agradables y cómodas.

21 Los servicios adicionales como la biblioteca, secretaría, cafetería, gimnasio y centro médico se prestan según lo prometido.

22 El personal administrativo y operativo refleja una apariencia pulcra.

23 El personal de logística está pendiente en la organización de los salones de clases y entrega de material de estudio

24 La universidad muestra un interés sincero en la formación integral y personal del estudiante

Apoyo institucional

25 Los horarios de clase son flexible para cumplir sus labores profesionales.

26 Alianza con universidades locales e internacionales

27 Los plazos o las formas de pago que ofrece la institución educativa

28 Los programas de pasantías a nivel internacional 